\title{
Neuroprotective Effects of Stiripentol on Oxygen-Glucose Deprivation in Primary Culture of Fetal Mice Cortical Neurons
}

\section{Noorolhoda Fotovat Eskandari ${ }^{1}$, Gelareh Vahabzadeh ${ }^{1,3 *}$, Fereshteh Golab ${ }^{1}$, Fariba Karimzadeh ${ }^{2}$, Parvaneh Rahimi-Moghadam ${ }^{3}$, Somayyeh Nasiripour ${ }^{4}$, Robabeh Shabani ${ }^{1}$}

${ }^{1}$ Cellular and Molecular Research Center, Iran University of Medical Sciences, Tehran, Iran

${ }^{2}$ Shefa Neuroscience Research Center, Khatam Alanbia Hospital, Tehran, Iran

${ }^{3}$ Department of Pharmacology, School of Medicine, Iran University of Medical Sciences, Tehran, Iran

${ }^{4}$ Department of Clinical Pharmacy, School of Medicine, Iran University of Medical Sciences, Tehran, Iran

\section{ABSTRACT}

Introduction: Excessive activation of NMDA receptors in ischemic injury as well as reduction of GABAergic system leads to discrepancies of ionic homeostasis and neuronal death. The aim of the present study was to evaluate the effect of different concentrations of stiripentol $(0.01$, $0.1,1,5,10,30 \mu \mathrm{M})$ as a GABAA receptor agonist on primary cortical culture of mice on $4 \mathrm{~h}$ oxygen-glucose deprivation (OGD). Materials and Methods: After 24h of incubation of neuronal cells with stiripentol, the cells were transferred to glucose-free DMEM (Dulbecco's Modified Eagle Medium) and were exposed to $4 \mathrm{~h}$ hypoxia in a small anaerobic chamber and incubated in standard condition for $24 \mathrm{~h}$. Cell viability was evaluated by MTT assay. Results: The results showed that different concentrations of stiripentol could increase the cell viability after 4h OGD Recovery (OGD/R). However, the protective effect of stiripentol was lower than the control group (the cells did not expose to OGD/R). Conclusion: Our results indicated that stiripentol could be a potential drug for treatment of brain ischemic condition. However, additional studies are needed to evaluate the mechanims of strripentol effect.

\section{Key words:}

1. gamma-Aminobutyric Acid

2. Ischemia

3. Mice

*Corresponding Author: Gelareh Vahabzadeh

E-mail: Gelareh.Vahabzadeh@gmail.com

doi: 10.18869/acadpub.shefa.5.1.10 


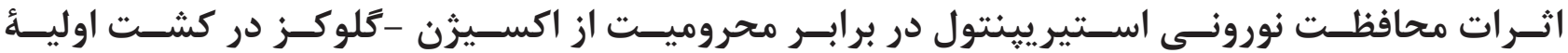

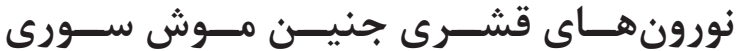

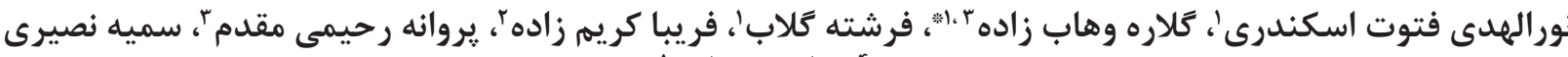

$$
\begin{aligned}
& \text { يور "، ربابه شعبانى } \\
& \text { 'مركز تحقيقات سلولى و مولكولى، دانشعاه علوم يزشكى ايران، تهران، ايران } \\
& \text { 'مركز تحقيقات علوم اعصاب شفا، بيمارستان خاتم الانبياء، تهران، ايران } \\
& \text { rتروه فارماكولوزى، دانشكده يزشكى، دانشخاه علوم يزشكى ايران، تهران، ايران }
\end{aligned}
$$

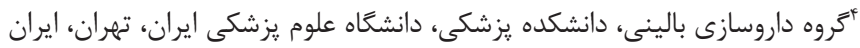

\section{اطلاعات مقاله:}

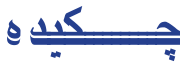

مقدمه: فعاليت بيش از حد كيرندههاى NMDA در آسيب ايسكمى و همجنين كاهش سيستهم Fابا منجر

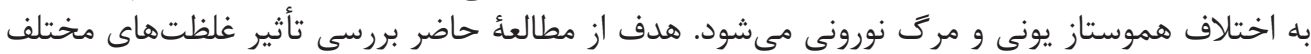

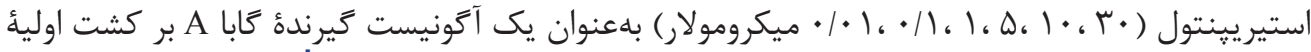

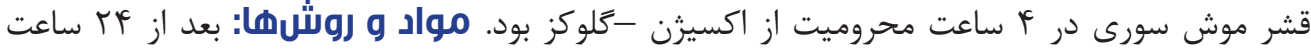

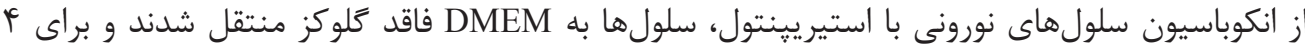

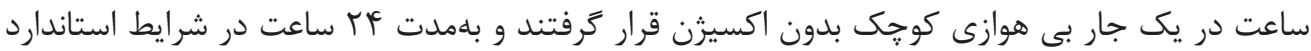

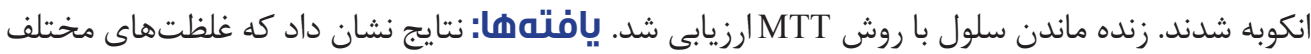

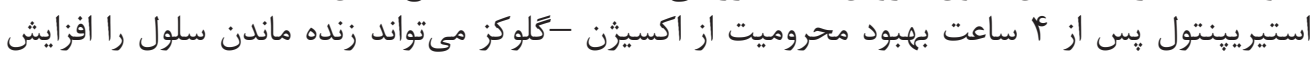

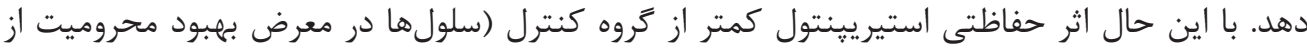

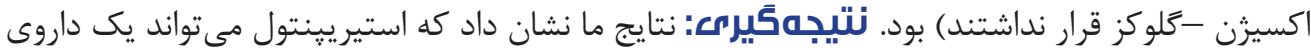

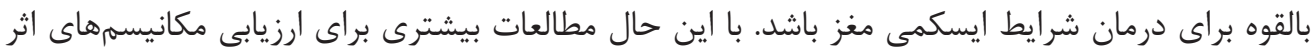
استيريينتول مورد نياز است.

كليد وازهها:

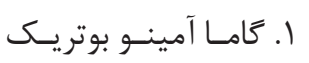

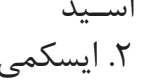

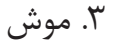

" نويسنده مسئول: كلاره وهاب زاده

آدرس الكترونيكى: Gelareh.Vahabzadeh@gmail.com 


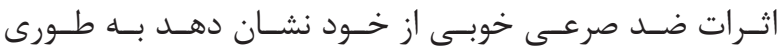

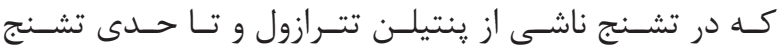

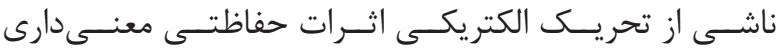

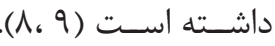

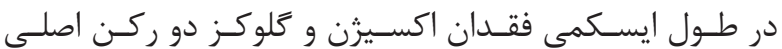

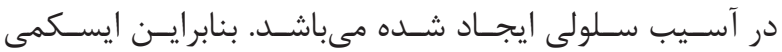

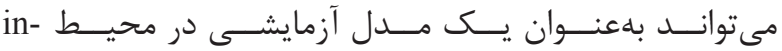

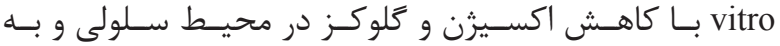

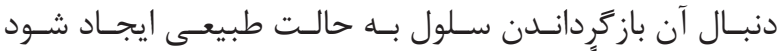

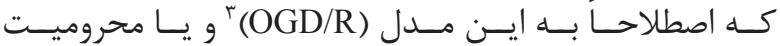

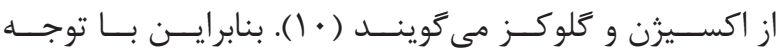

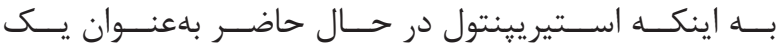

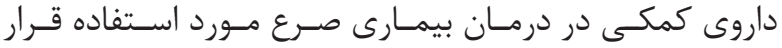

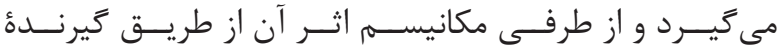

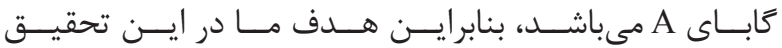

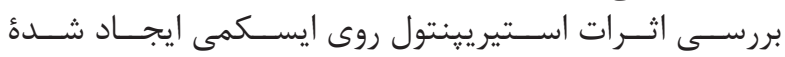

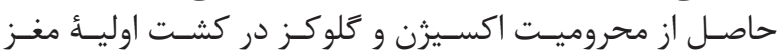

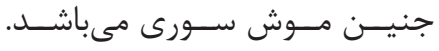

مواد و روشها

تهيئ كشت اوليةُ نورونى از قشر مغز جنين موش سورى

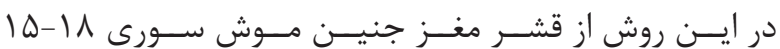

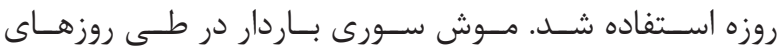

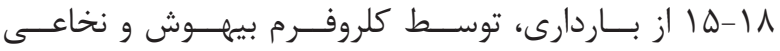

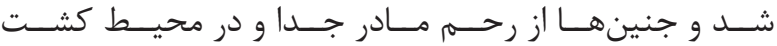

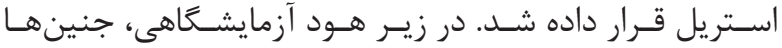

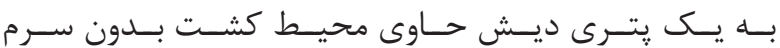

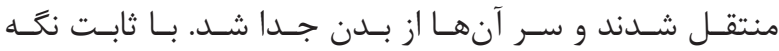

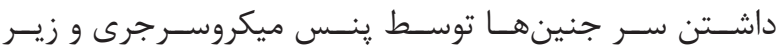
ميكروسـكو

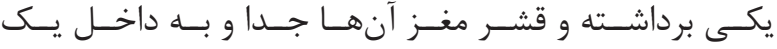

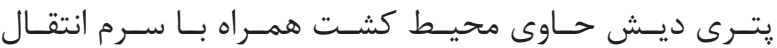

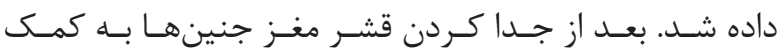

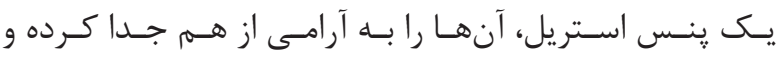

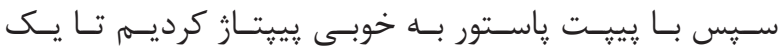

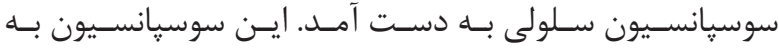

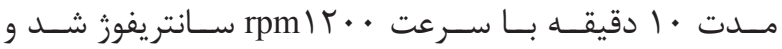

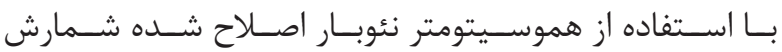

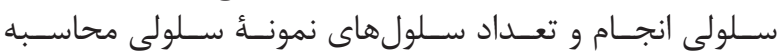

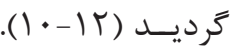

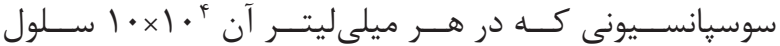

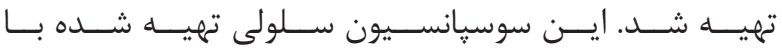

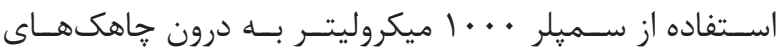

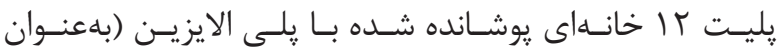

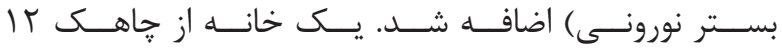

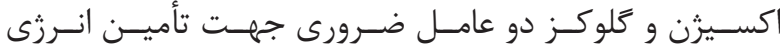

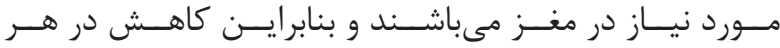

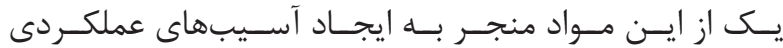

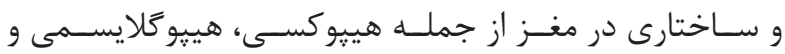

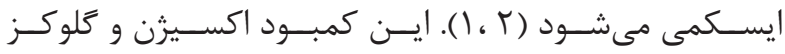

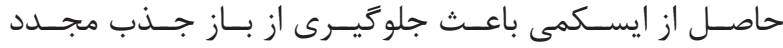

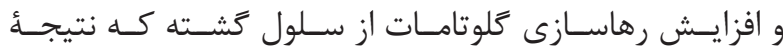

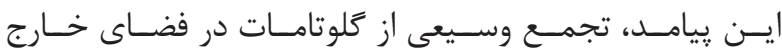

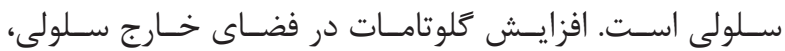

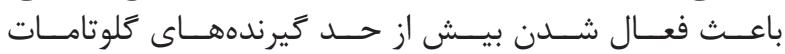

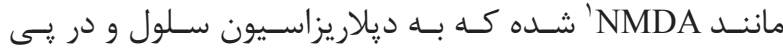

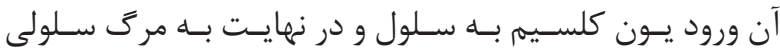

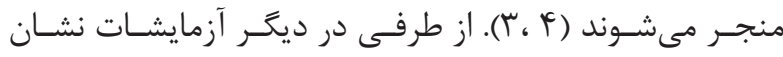

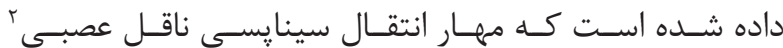

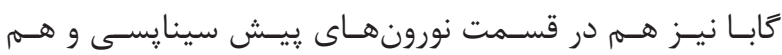

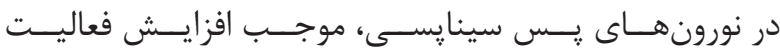

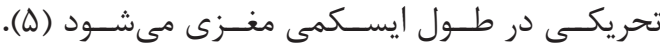

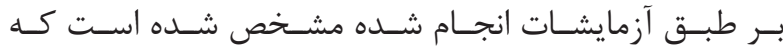

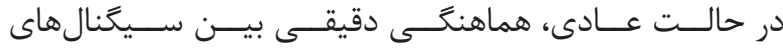

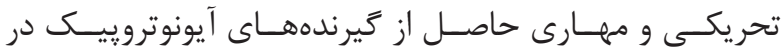

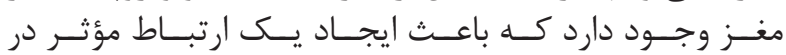

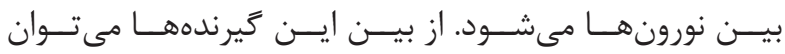

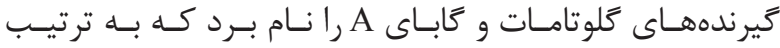

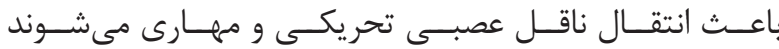

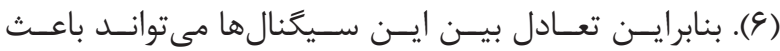

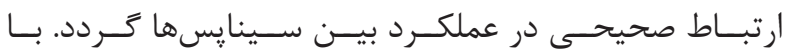

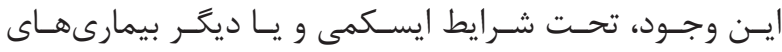

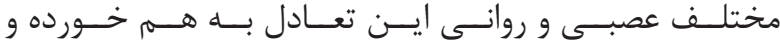

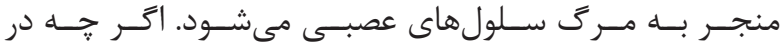

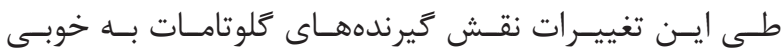

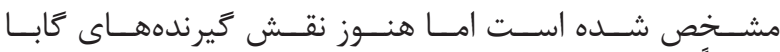

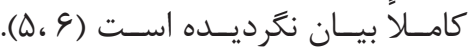

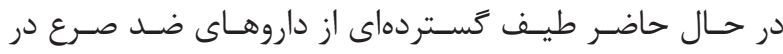

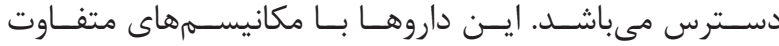

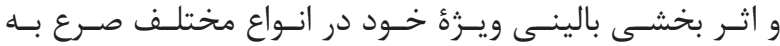

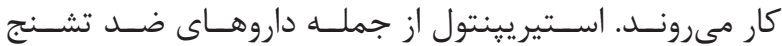

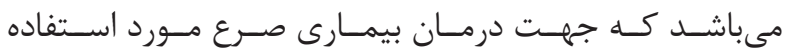

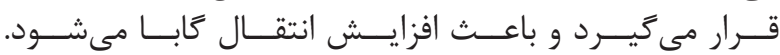

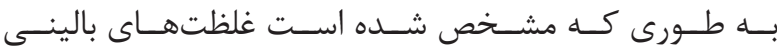

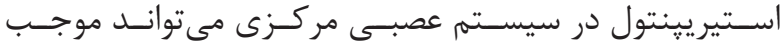

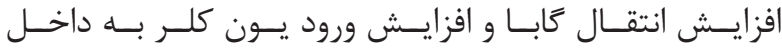

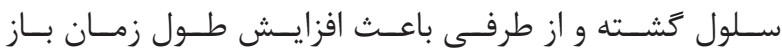

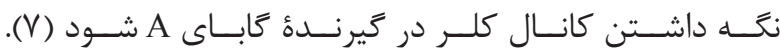

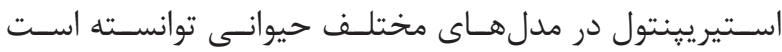




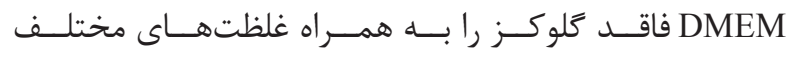

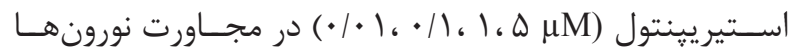

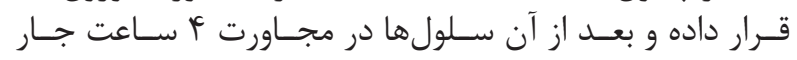

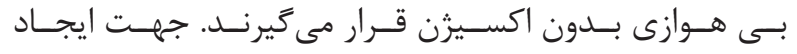

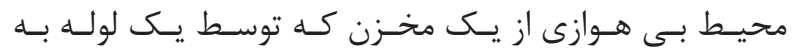

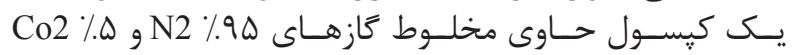

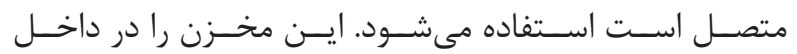

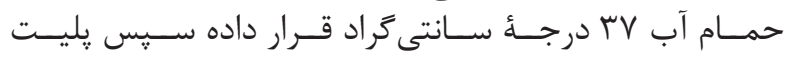

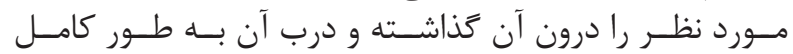

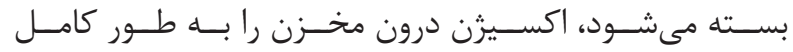

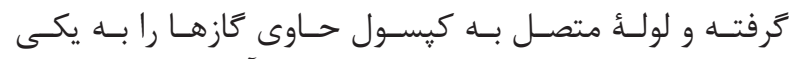

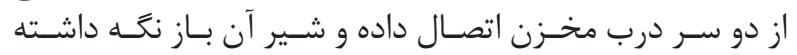

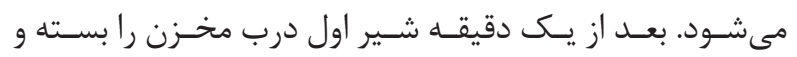

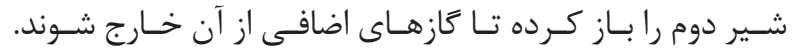

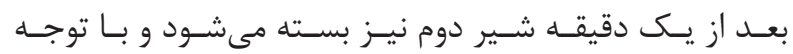

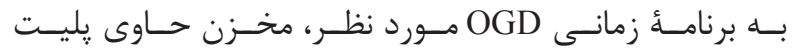

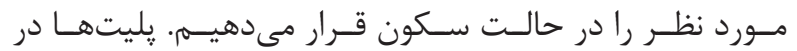

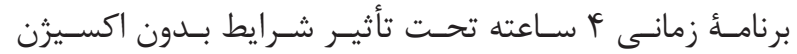

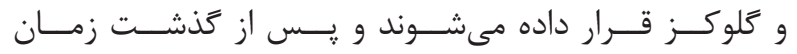

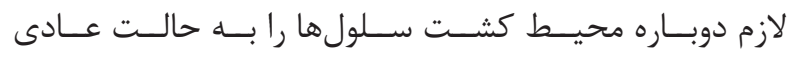

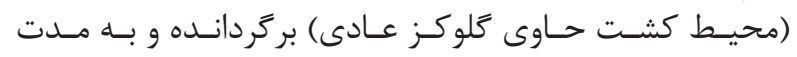

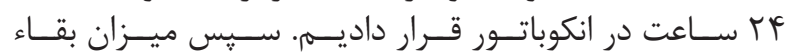

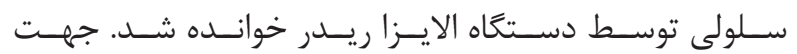

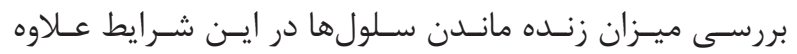

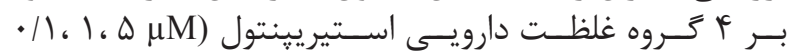

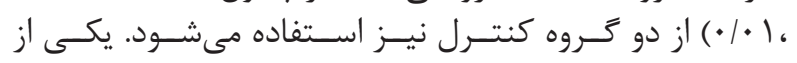

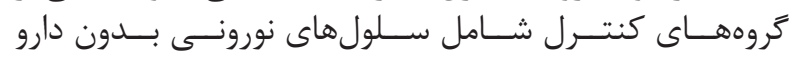

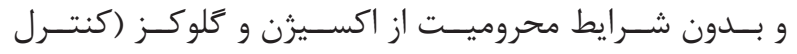

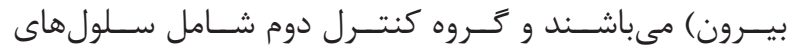

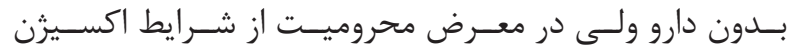

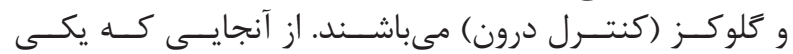

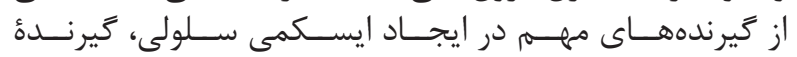

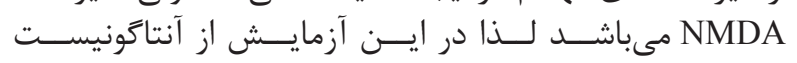
ايسن زيرنـده بـهـ نـام

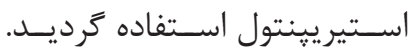

بررسى ميزان زنده بودن سلولها با استفاده از آزمون

MTT

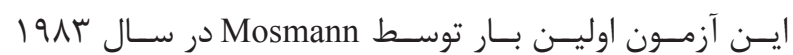

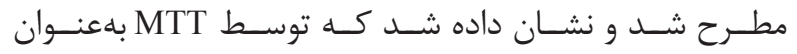

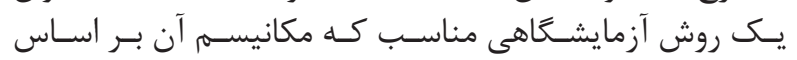

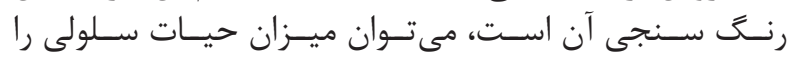

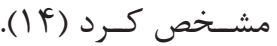

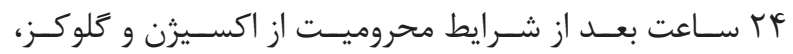

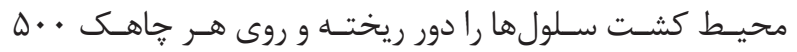

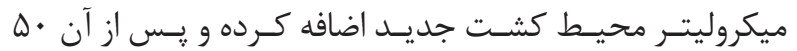

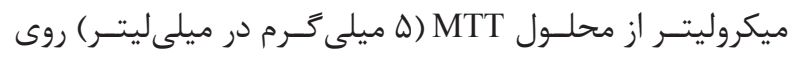

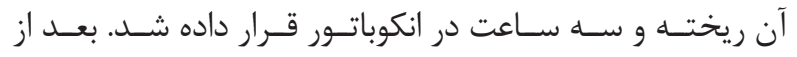

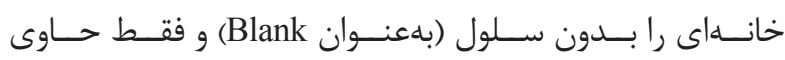

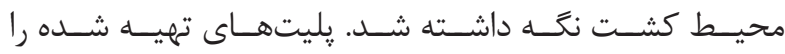

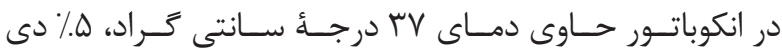

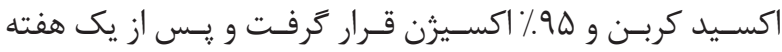

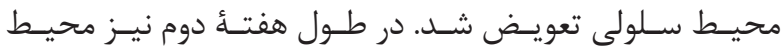

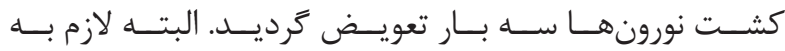

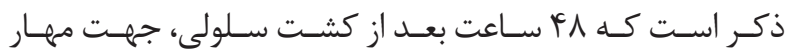

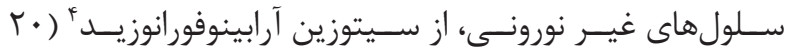

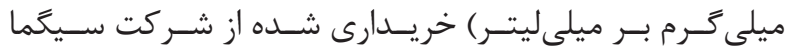

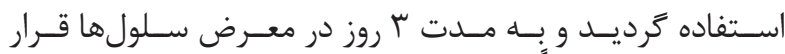

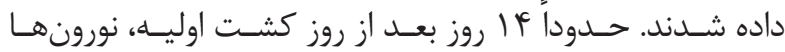

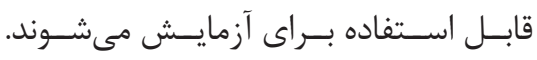

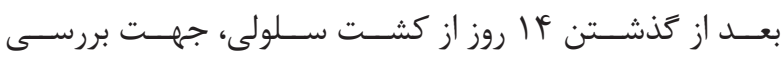

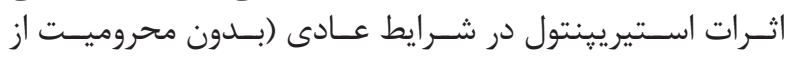

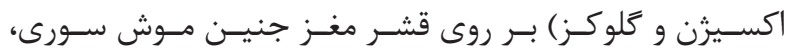

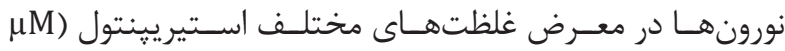

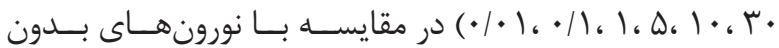

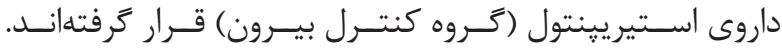

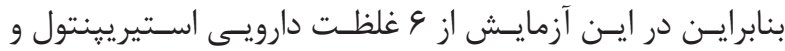

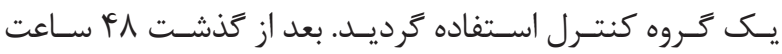

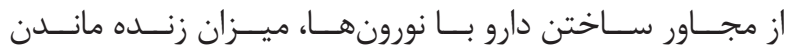

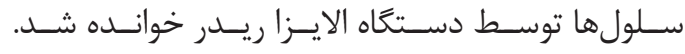

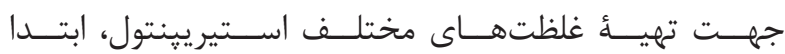

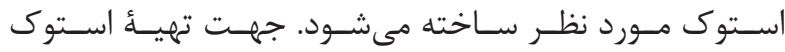

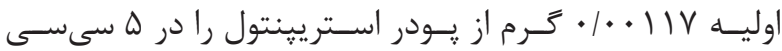
DMSO

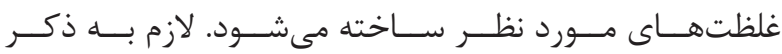

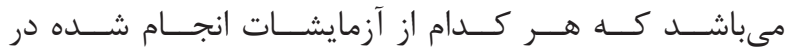

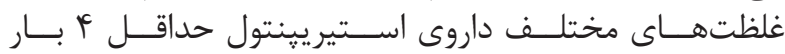
تكـــرار شـده اسـت مختلف

ايجاد مدل ايسكمى در محيط in vitro

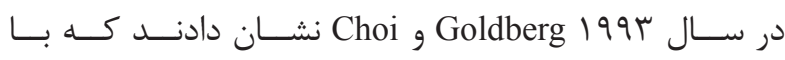

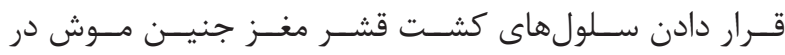

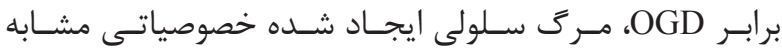

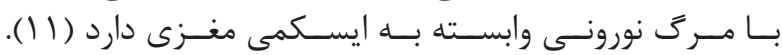

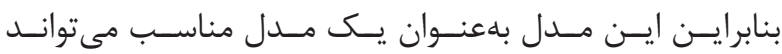

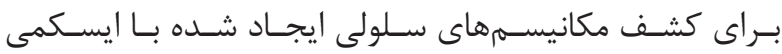

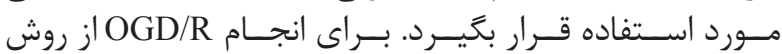

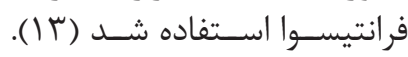

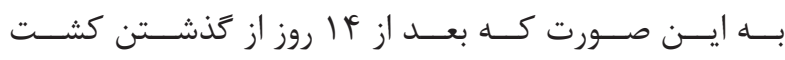

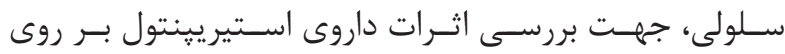

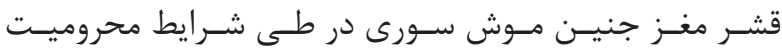

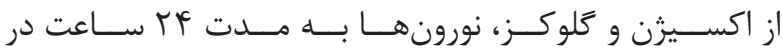

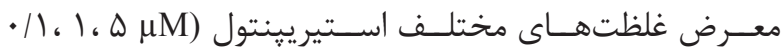

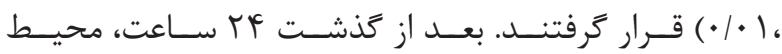

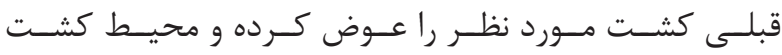

${ }^{4}$ Cytosine 1- $\beta$-D-arabinoforanoside 


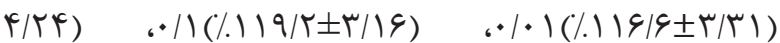
معن

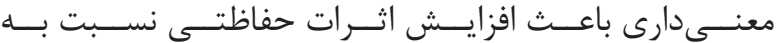

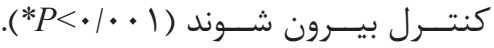

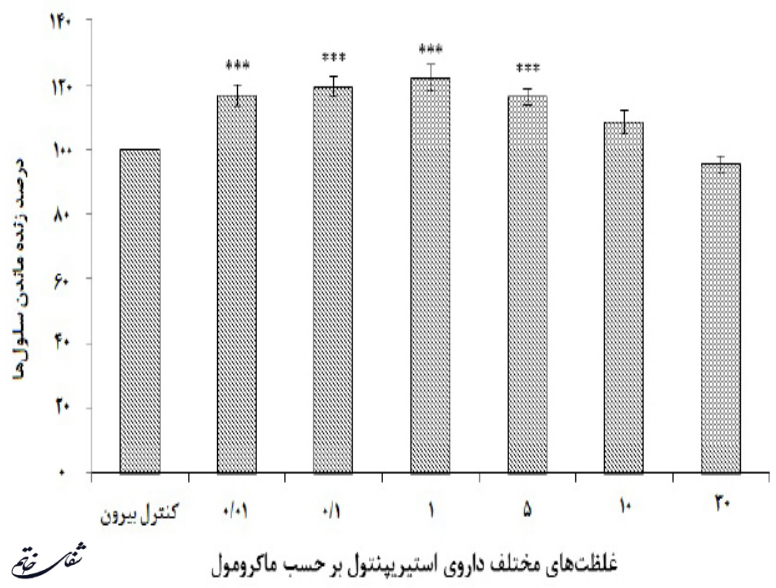

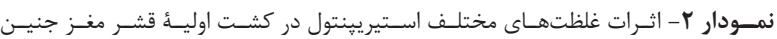

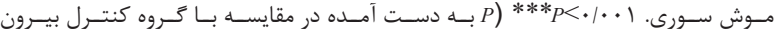

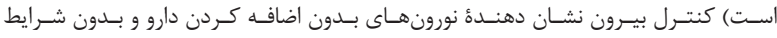

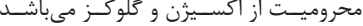

بررسى ميزان زنده بودن كشت نورونهاى قشر مغز

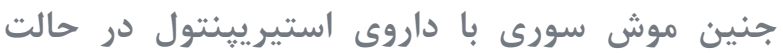
شر ايط محروميت از اكسيثرن و تلوكز

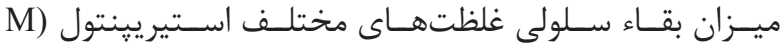

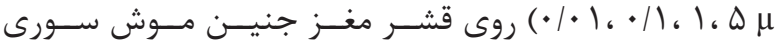

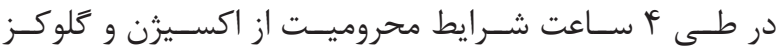

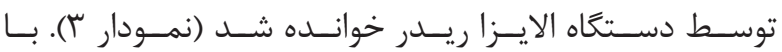

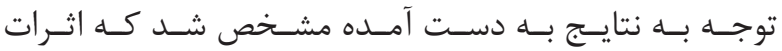

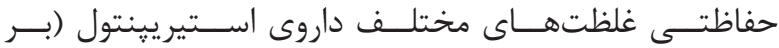

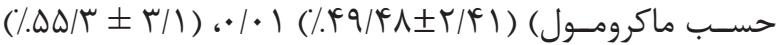

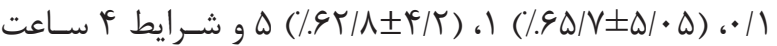

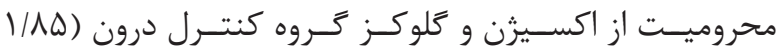
土.

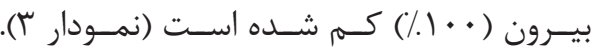

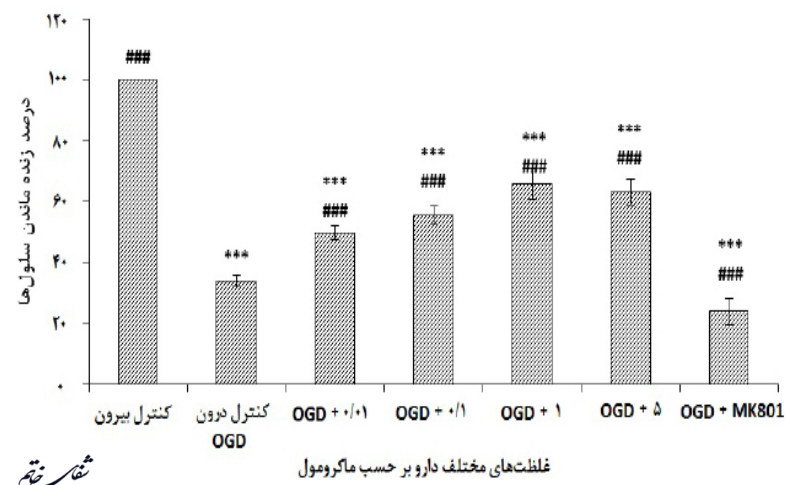

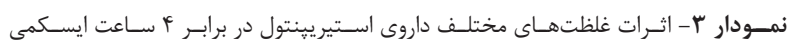

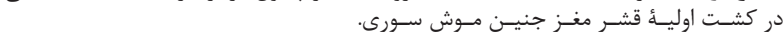

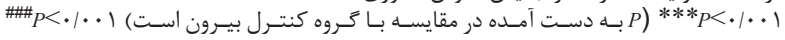

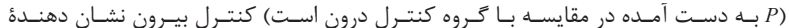

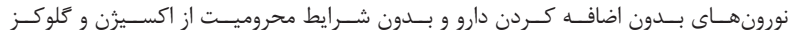

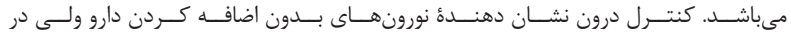

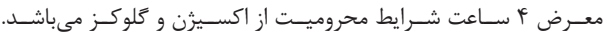

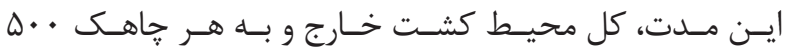

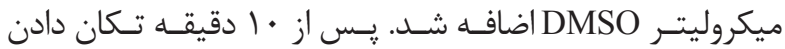

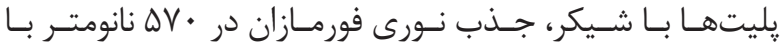

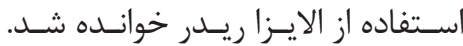

تجزيه و تحليل دادهها

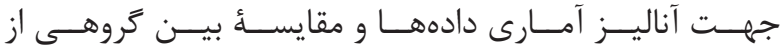

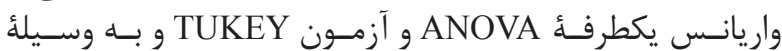

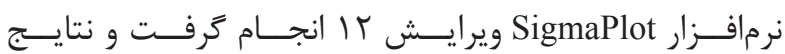

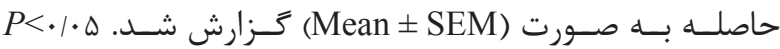

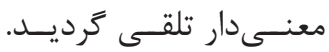

يافتهها

بررسى اثر محروميت از اكسيثزن و كلوكز روى كشت اولية

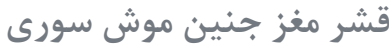

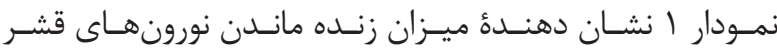

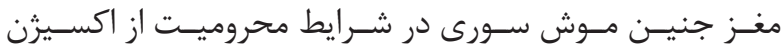

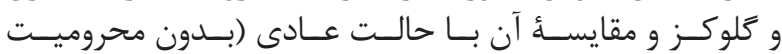

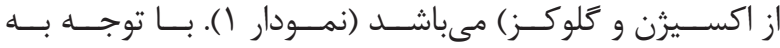

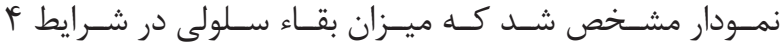

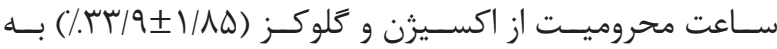

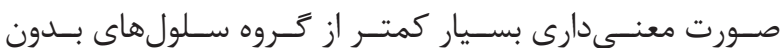

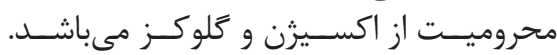

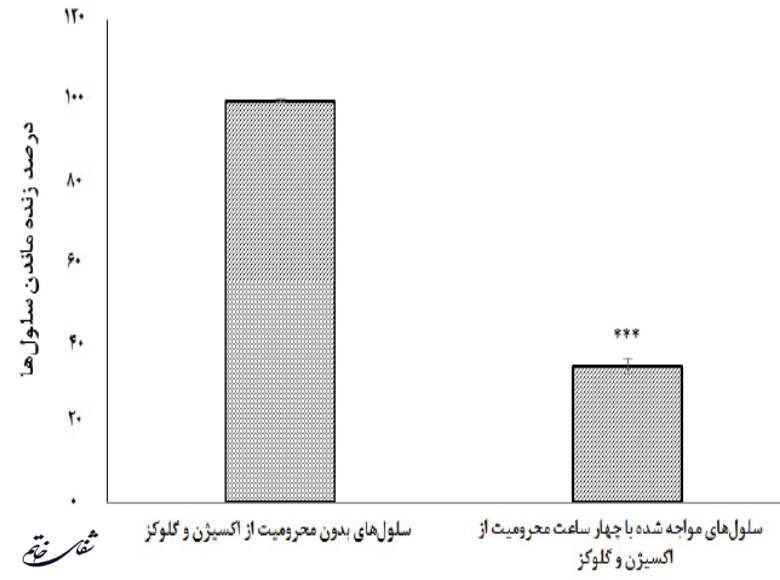

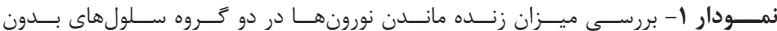

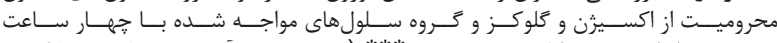

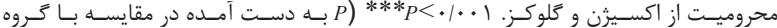

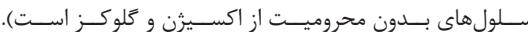

بررسى ميزان زنده بودن كشت نورونهاى قشر مغز جنين

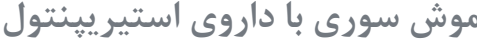

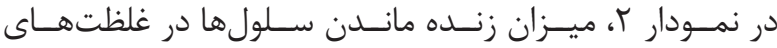

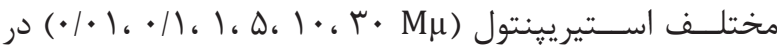

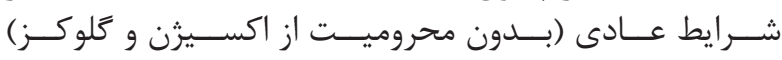

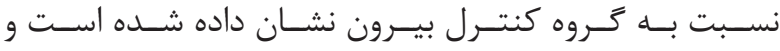

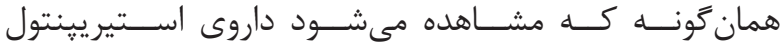

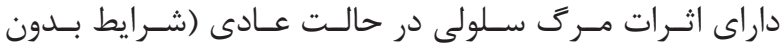

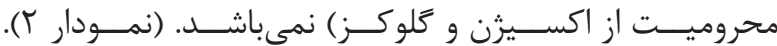

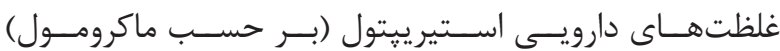




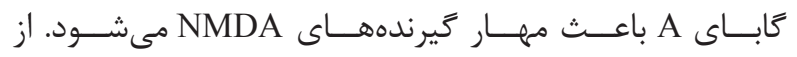

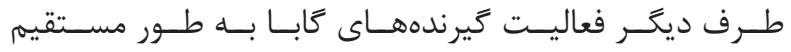

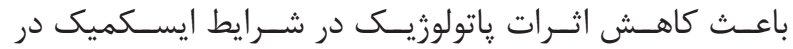

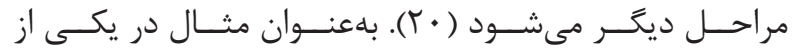

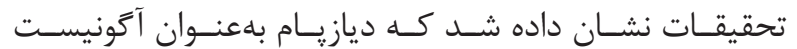

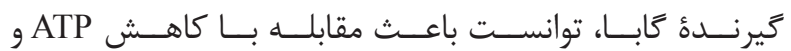

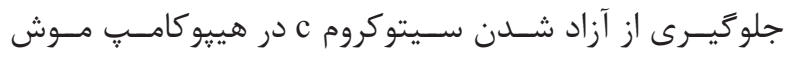

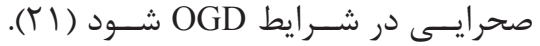

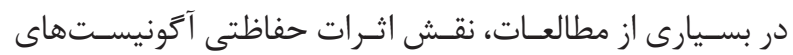

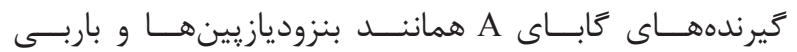

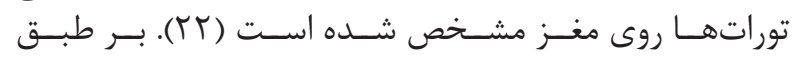

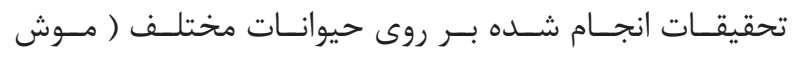

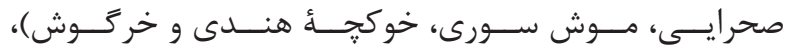

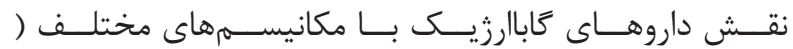

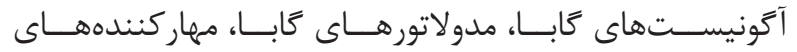

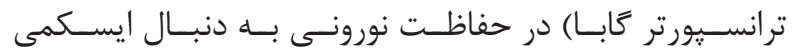

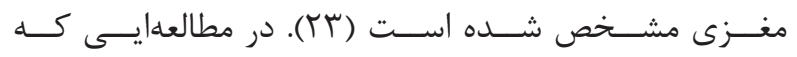

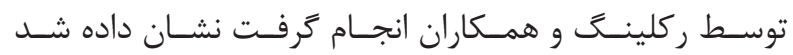

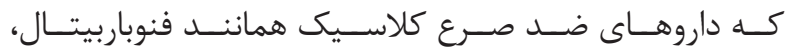

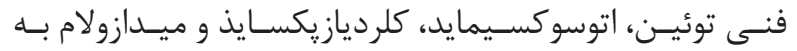

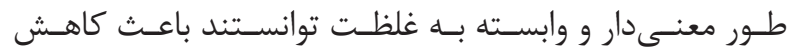

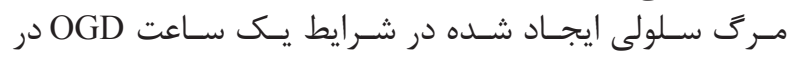

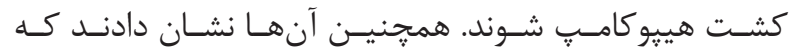

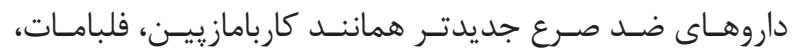

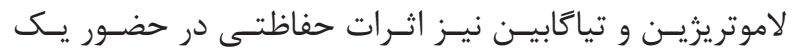

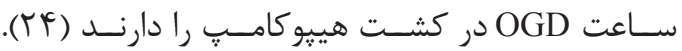

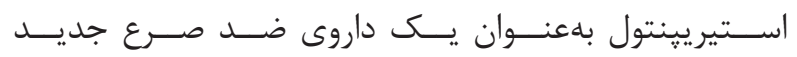

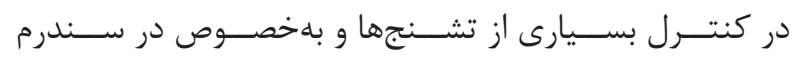

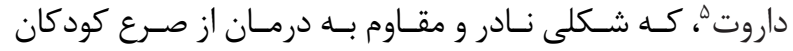

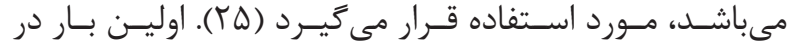

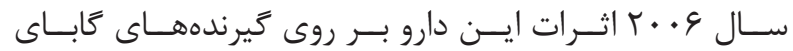

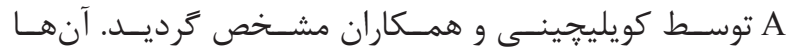

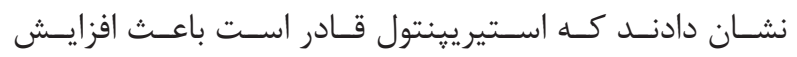

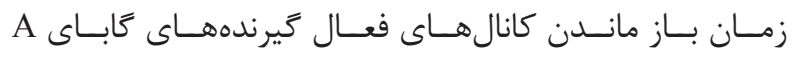

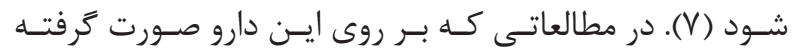

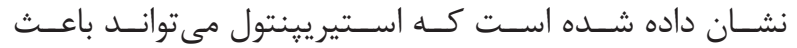

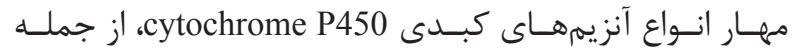

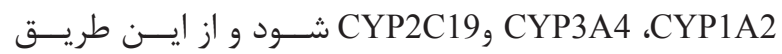

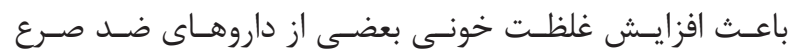

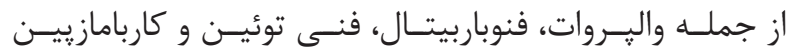

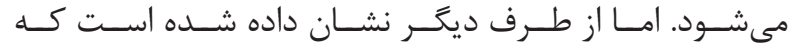

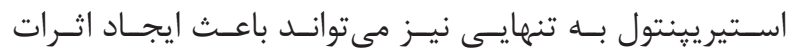

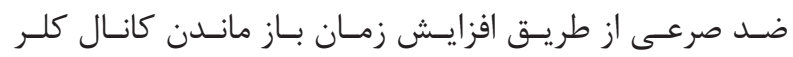

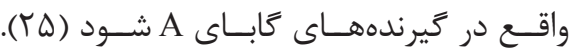

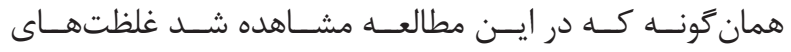

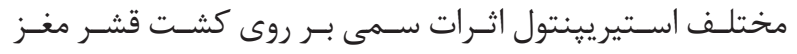

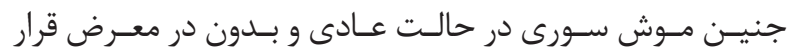

بحث و نتيجهَ

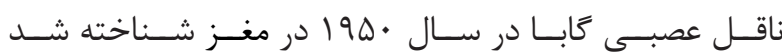

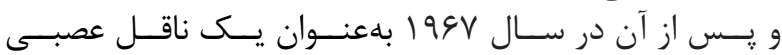

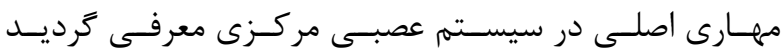

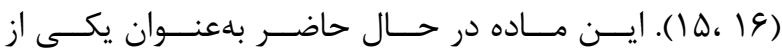

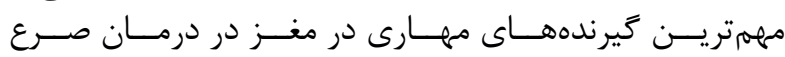

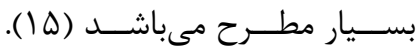

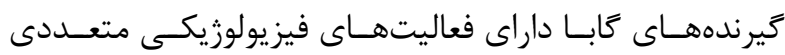

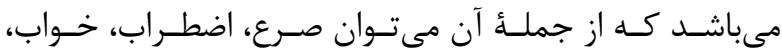

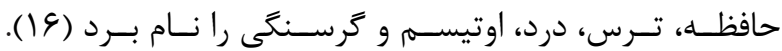

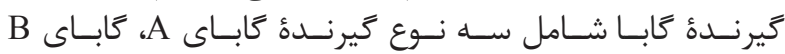

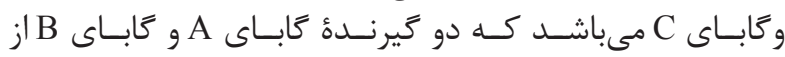

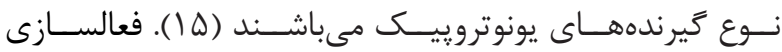

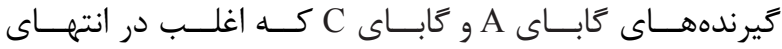

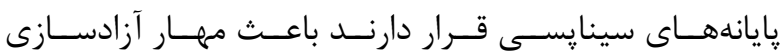

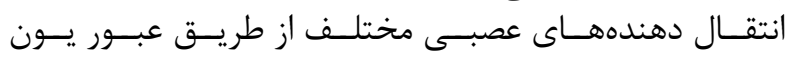

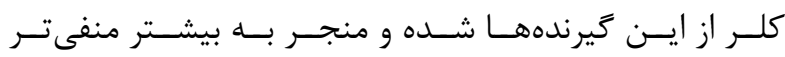

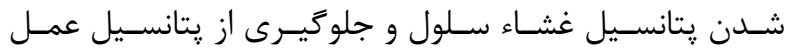
مىشـود (9) (9).

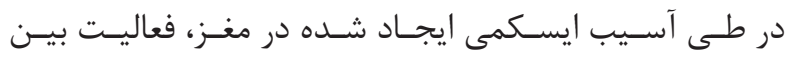

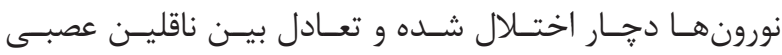

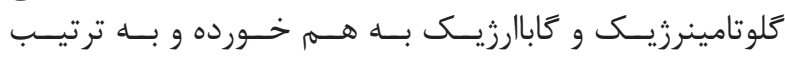

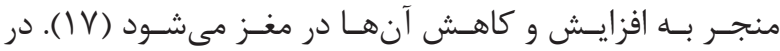

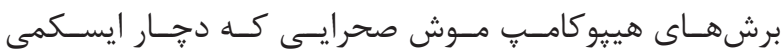

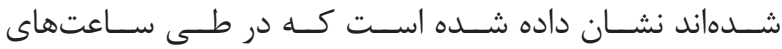

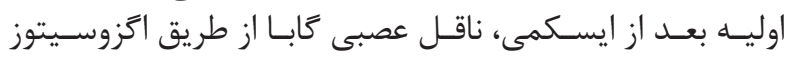

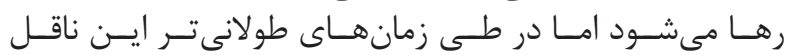

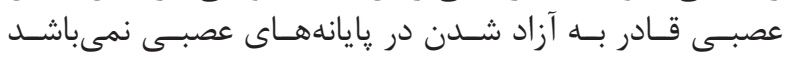

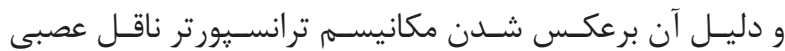

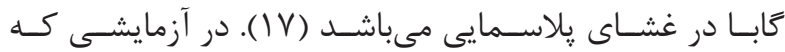

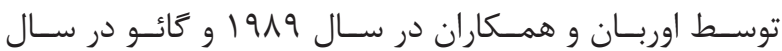

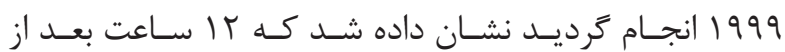

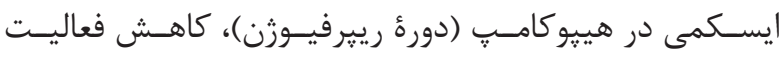

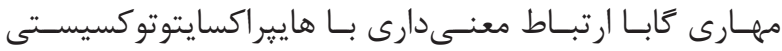

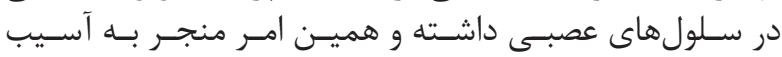

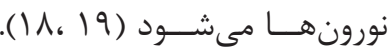

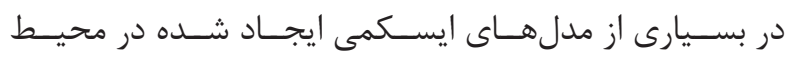
in vivo in vitro

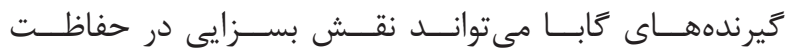

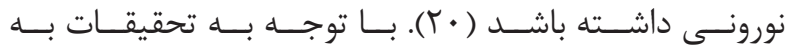

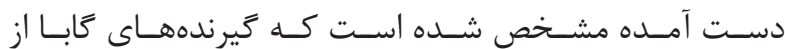

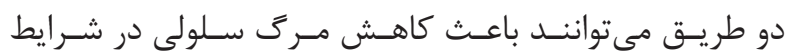

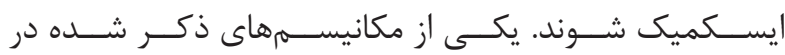

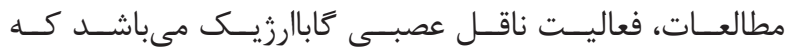

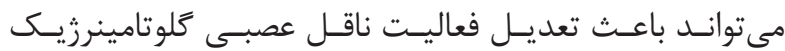

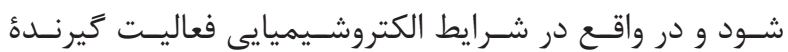




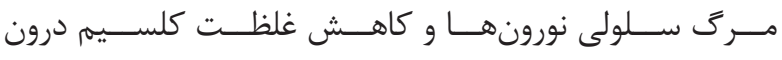

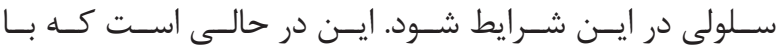

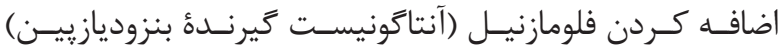

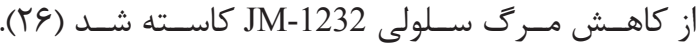

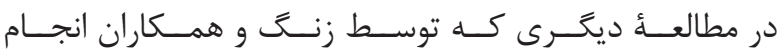

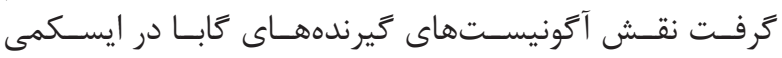

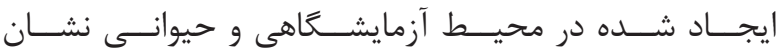

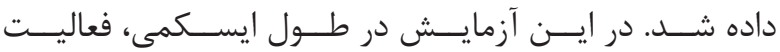

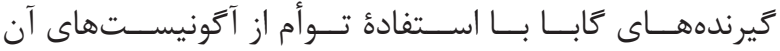

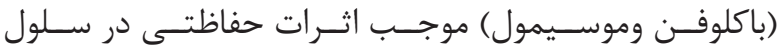

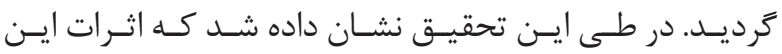

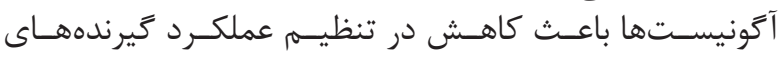

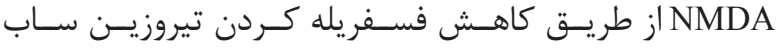

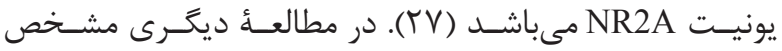

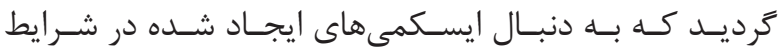
iv vivo

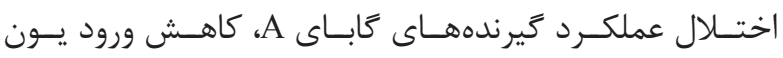

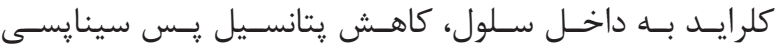

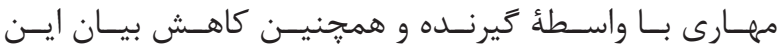

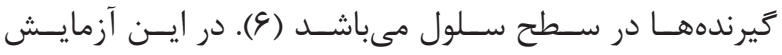

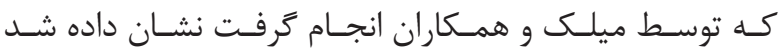

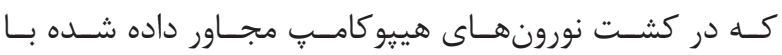

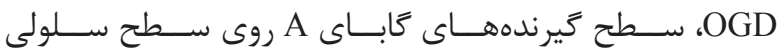

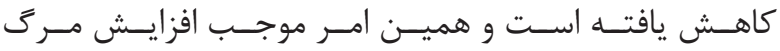

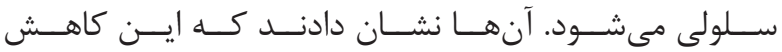

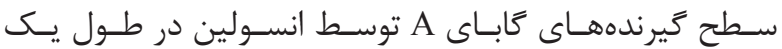

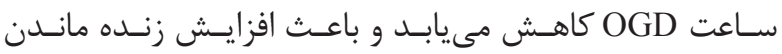

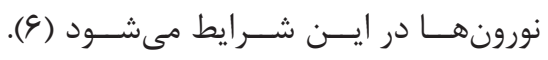

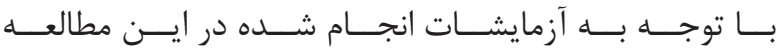

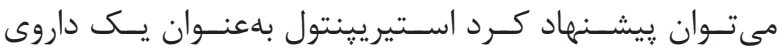

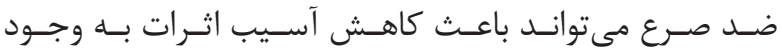

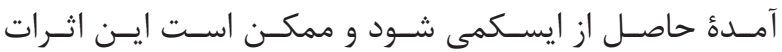

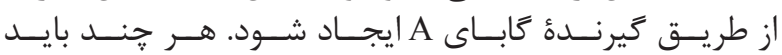

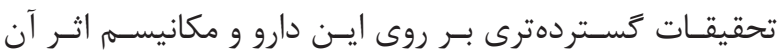

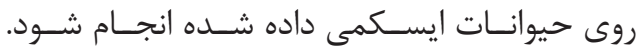

1. Tian GF, Baker AJ. Protective effect of high glucose against ischemia-induced synaptic transmission damage in rat hippocampal slices. J Neurophysiol. 2002; 88(1): 236-48.

2. Vahabzadeh G, Ebrahimi S-A, Rahbar-Roshandel N, Mahmoudian MM. The effect of noscapine on oxygenglucose deprivation on primary murine cortical neurons in high glucose condition. Iran J Pharm Res. 2016; 15(2): 501-12.

3. Harukuni I, Bhardwaj A. Mechanisms of brain injury after global cerebral ischemia. Neurol Clin. 2006; 24(1): 1-21.

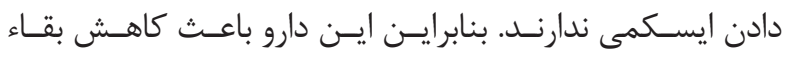

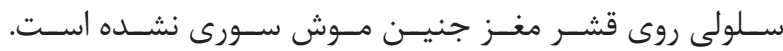

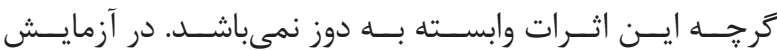

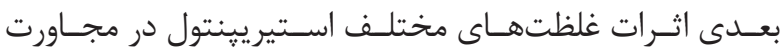

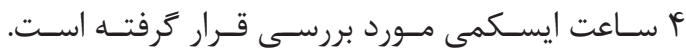

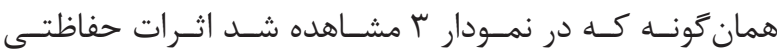

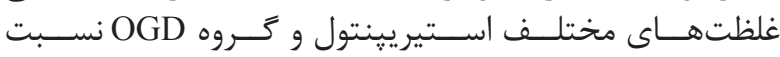

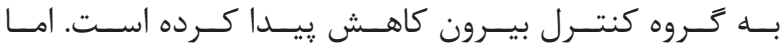

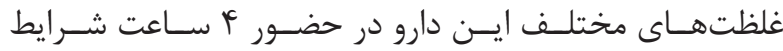

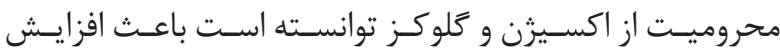

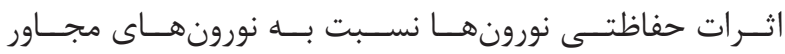

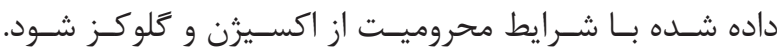

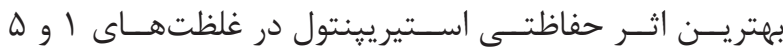

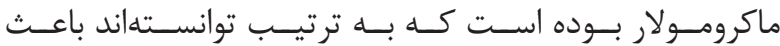

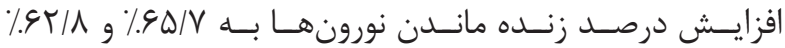

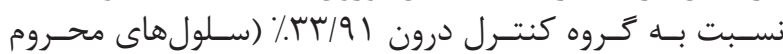

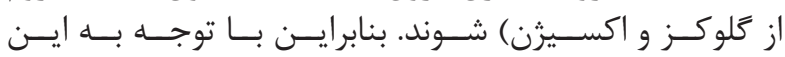

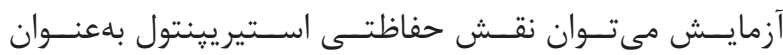

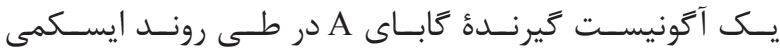

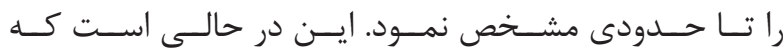

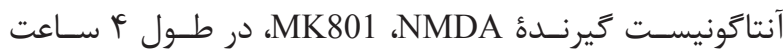

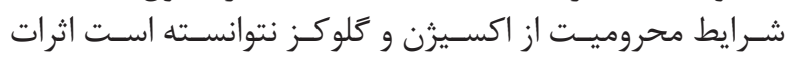

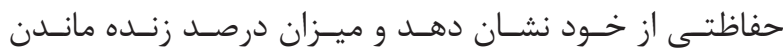

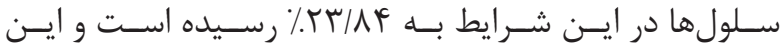

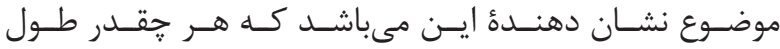

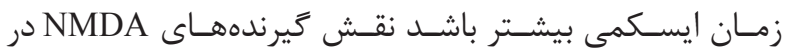

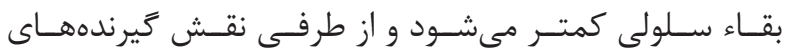

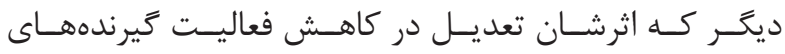
NMDA

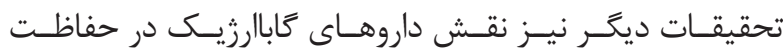

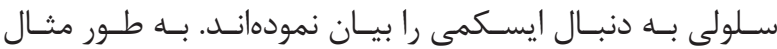

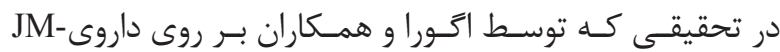

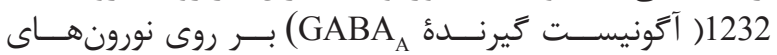

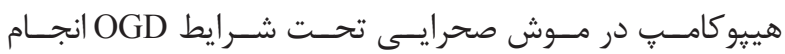

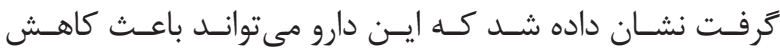

منابع

4. Dennis SH, Jaafari N, Cimarosti H, Hanley JG, Henley JM, Mellor JR. Oxygen/glucose deprivation induces a reduction in synaptic AMPA receptors on hippocampal CA3 neurons mediated by mGluR1 and adenosine A3 receptors. J Neurosci. 2011; 31(33): 11941-52.

5. Mele M, Aspromonte MC, Duarte CB. Downregulation of GABAA receptor recycling mediated by HAP1 contributes to neuronal death in in vitro brain ischemia. Mol Neurobiol. 2016: 1-13.

6. Mielke JG, Wang YT. Insulin exerts neuroprotection by counteracting the decrease in cell-surface GABAA receptors following oxygen-glucose deprivation in 
cultured cortical neurons. J Neurochem. 2005; 92(1): 103-13.

7. Quilichini PP, Chiron C, Ben-Ari Y, Gozlan H. Stiripentol, a putative antiepileptic drug, enhances the duration of opening of GABAA-receptor channels. Epilepsia. 2006; 47(4): 704-16.

8. Poisson M, Huguet F, Savattier A, Bakri-Logeais F, Narcisse G. A new type of anticonvulsant, stiripentol. pharmacological profile and neurochemical study. Arzneimittel-Forsch. 1984; 34(2): 199-204.

9. Shen DD, Levy R, Moor MJ, Savitch JL. Efficacy of stiripentol in the intravenous pentylenetetrazol infusion seizure model in the rat. Epilepsy Res. 1990; 7(1): 40-8.

10. Vahabzadeh G, Rahbar-Roshandel N, Ebrahimi S-A, Mahmoudian M. Neuroprotective effect of noscapine on cerebral oxygen-glucose deprivation injury. Pharmacol Rep. 2015; 67(2): 281-8.

11. Goldberg MP, Choi DW. Combined oxygen and glucose deprivation in cortical cell culture: calciumdependent and calcium-independent mechanisms of neuronal injury. J Neurosci. 1993; 13(8): 3510-24.

12. Dawson VL, Dawson TM, London ED, Bredt DS, Snyder SH. Nitric oxide mediates glutamate neurotoxicity in primary cortical cultures. Proc Natl Acad Sci. 1991; 88(14): 6368-71.

13. Frantseva MV, Carlen PL, El-Beheiry H. A submersion method to induce hypoxic damage in organotypic hippocampal cultures. J Neurosci Meth. 1999; 89(1): 25-31.

14. Mosmann T. Rapid colorimetric assay for cellular growth and survival: application to proliferation and cytotoxicity assays. J Immunol Methods. 1983; 65(1): 55-63.

15. Llorente IL, Perez-Rodriguez D, MartínezVillayandre B, Dos-Anjos S, Darlison MG, Poole AV, et al. GABA A receptor chloride channels are involved in the neuroprotective role of GABA following oxygen and glucose deprivation in the rat cerebral cortex but not in the hippocampus. Brain Res. 2013; 1533: 141-51.

16. Rashidi A, Ahmadi S. Subunits of gammaaminobutyric acid receptors and their roles in neuropsychological disorders. Shefaye Khatam. 2014; 2(2): $70-80$.
17. Mele M, Ribeiro L, Inácio AR, Wieloch T, Duarte CB. GABA A receptor dephosphorylation followed by internalization is coupled to neuronal death in in vitro ischemia. Neurobiol Dis. 2014; 65: 220-32.

18. Gao T, Pulsinelli W, Xu Z. Changes in membrane properties of CA1 pyramidal neurons after transient forebrain ischemia in vivo. Neuroscience. 1999; 90(3): 771-80.

19. Urban L, Neill K, Crain B, Nadler J, Somjen G. Postischemic synaptic physiology in area CA1 of the gerbil hippocampus studied in vitro. J Neurosci. 1989; 9(11): 3966-75.

20. Loetscher PD, Rossaint J, Rossaint R, Weis J, Fries M, Fahlenkamp A, et al. Argon: neuroprotection in in vitro models of cerebral ischemia and traumatic brain injury. Crit Care Med. 2009; 13(6): 1. doi: 10.1186/ cc8214.

21. Galeffi F, Sinnar S, Schwartz-Bloom RD. Diazepam promotes ATP recovery and prevents cytochrome c release in hippocampal slices after in vitro ischemia. $\mathrm{J}$ Neurochem. 2000; 75(3): 1242-9.

22. Bickler PE, Warner DS, Stratmann G, Schuyler JA. $\gamma$-Aminobutyric acid-a receptors contribute to isoflurane neuroprotection in organotypic hippocampal cultures. Anesth Analg. 2003; 97(2): 564-71.

23. Schwartz-Bloom RD, Sah R. $\gamma$-Aminobutyric acid A neurotransmission and cerebral ischemia. J Neurochem. 2001; 77(2): 353-71.

24. Rekling JC. Neuroprotective effects of anticonvulsants in rat hippocampal slice cultures exposed to oxygen/glucose deprivation. Neurosci Lett. 2003; 335(3): 167-70.

25. Fisher JL. The effects of stiripentol on GABAA receptors. Epilepsia. 2011; 52(s2): 76-8.

26. Ogura T, Hamada T, Matsui T, Tanaka S, Okabe S, Kazama T, et al. Neuroprotection by JM-1232 (-) against oxygen-glucose deprivation-induced injury in rat hippocampal slice culture. Brain Res. 2015; 1594: 52-60.

27. Zhang F, Li C, Wang R, Han D, Zhang Q-G, Zhou $\mathrm{C}$, et al. Activation of GABA receptors attenuates neuronal apoptosis through inhibiting the tyrosine phosphorylation of NR2A by Src after cerebral ischemia and reperfusion. Neuroscience. 2007; 150(4): 938-49. 